\title{
Nitidine chloride inhibits hepatocellular carcinoma cell growth in vivo through the suppression of the JAK1/STAT3 signaling pathway
}

\author{
JUN LIAO $^{1 *}$, TENG XU ${ }^{2 *}$, JIA-XUAN ZHENG ${ }^{1}$, JIU-MAO LIN $^{2}$, \\ QIAO-YAN CAI ${ }^{2}$, DE-BIAO YU ${ }^{1}$ and JUN PENG ${ }^{2}$ \\ ${ }^{1}$ Department of Acupuncture and Moxibustion, ${ }^{2}$ Academy of Integrative Medicine Biomedical Research Center, \\ Fujian University of Traditional Chinese Medicine, Fuzhou, Fujian 350122, P.R. China
}

Received February 22, 2013; Accepted April 15, 2013

DOI: $10.3892 / \mathrm{ijmm} .2013 .1358$

\begin{abstract}
Signal transducer and activator of transcription 3 (STAT3) is persistently activated in cancer cells and contributes to malignant progression in various types of cancer. The Janus-activated kinase (JAK) family phosphorylates STAT3 in response to stimulation by cytokines or growth factors. The JAK1-STAT3 signaling pathway plays an important role in cell proliferation and apoptosis. Nitidine chloride (NC) is a benzophenanthridine alkaloid that has been reported as an antitumor agent due to its its inhibitory effects on topoisomerase I. Using a mouse xenograft model of hepatocellular carcinoma (HCC), this study aimed to evaluate the effects of $\mathrm{NC}$ on tumor growth in vivo and to elucidate the underlying mechanisms. The analysis of the effects of NC on apoptosis in HCC tumor xenografts in mice was carried out by terminal deoxynucleotidyl transferase-mediated dUTP nick end-labeling (TUNEL) assay; the expression of Bcl-2, Bax, cyclin-dependent kinase (CDK)4,
\end{abstract}

Correspondence to: Dr Jun Liao, Department of Acupuncture and Moxibustion, Fujian University of Traditional Chinese Medicine, 1 Huatuo Road, Minhou Shangjie, Fuzhou, Fujian 350122, P.R. China E-mail: 77liaojun@163.com

Dr Jun Peng, Academy of Integrative Medicine Biomedical Research Center, Fujian University of Traditional Chinese Medicine, 1 Huatuo Road, Minhou Shangjie, Fuzhou, Fujian 350122, P.R. China

E-mail: pjunlab@hotmail.com

*Contributed equally

Abbreviations: HCC, hepatocellular carcinoma; JAK1, Janus-activated kinase 1; STAT3, signal transducer and activator of transcription 3; NC, nitidinechloride; TUNEL, terminaldeoxynucleotidyl transferasemediated dUTP nick end-labeling; PCNA, proliferating cell nuclear antigen

Key words: nitidine chloride, Chinese medicine, hepatocellular carcinoma, Janus-activated kinase 1 , signal transducer and activator of transcription 3, apoptosis, proliferation cyclin D1, p21 and proliferating cell nuclear antigen (PCNA) was analyzed by immunohistochemistry; and the protein expression of JAK1 and STAT3 was examined by western blot analysis. Our results revealed that treatment with $\mathrm{NC}$ decreased the tumor volume and tumor weight, suggesting that $\mathrm{NC}$ inhibits $\mathrm{HCC}$ cell growth in vivo. In addition, NC blocked the activation of JAK1-STAT3 in the tumor tissues, which in turn resulted in the induction of cancer cell apoptosis and the inhibition of proliferation. Consequently, treatment with NC downregulated the expression of cyclin D1, CDK4 and Bcl-2 and increased the level of p21 and Bax. Our data provide a molecular basis for the antitumor activity of NC.

\section{Introduction}

Hepatocellular carcinoma (HCC) is the third leading cause of cancer-related mortality worldwide $(1,2)$ and it has been reported that more than 600,000 individuals succumb to the disease each year (1). It is the second most common cause of cancer-related mortality in China, and $75 \%$ of known new cases and deaths in the Asia-Pacific region (3-5). Currently, the main treatment methods for liver cancer include surgical resection, radiotherapy and chemotherapy $(4,6)$. Although surgical resection (which involves removing the tumor completely) offers the best prognosis for long-term survival, only 10-15\% of patients are suitable for surgical resection, as the tumor may be too large, or may have grown into major blood vessels or other vital organs (7-9). Related data demonstrate that the percentage of HCC cells is already high at diagnosis with a high expression of the multidrug resistance gene and conventional chemotherapy of HCC fails to provide satisfactory remission and may cause serious side-effects $(6,10)$. Thus, it is necessary to develop a novel effective drug for the treatment of HCC. Natural products have attracted much attention in the search for novel anticancer therapeutic agents as they have relatively few side-effects and have long been used as alternative therapies for various diseases, including cancer $(11,12)$. Therefore, determining naturally occurring agents is a promising approach for anticancer treatment.

The Janus-activated kinase (JAK) family includes JAK1, JAK2, JAK3 and tyrosine kinase 2 (TYK2) (13). The JAK 
family activate signal transducer and activator of transcription (STAT) proteins in response to different cytokines and growth factors (14-17). In normal cells, JAK/STAT signaling is tightly regulated. However, in cancer cells, it is persistently activated due to the aberrant activation of JAK family kinases or other tyrosine kinases (18). Among the JAK-STAT family members, JAK1-STAT3 plays an important role in cell proliferation and apoptosis (19). STAT3 can stimulate endothelial cell migration and differentiation and lead to tumor VEGF overproduction by direct transcriptional activation (20-22). The JAK1-STAT3 pathway plays a crucial role in cell survival, angiogenesis, immune evasion and inflammatory responses through the activation of STAT3, causing cyclin D1, cyclin-dependent kinase (CDK)4, Bcl-2, Bax and vascular endothelial growth factor gene transcription (23-26). Thus, the JAK1-STAT3 signaling pathway as a target for intervention is expected to become a novel method for the treatment of HCC.

Zanthoxylum nitidum, belonging to the family Rutaceae, is a medicinal herb widely distributed in Northeastern Asia. As a well-known traditional Chinese folk medicine, it is used to promote the flow of Qi, relieve pain, promote blood circulation and to remove blood stasis (27). Nitidine is found in the root of Zanthoxylum nitidum and several studies have demonstrated that nitidine exerts antitumor, anti-inflammatory, anti-leukemic and even anti-HIV effects $(28,29)$. Previous studies on nitidine chloride (NC) have focused on topoisomerase I poison-mediated cell toxicity $(30,31)$. $\mathrm{NC}$ has also been reported to be involved in the suppression of cell growth, the induction of cell apoptosis, cell cycle arrest and the inhibition of the activity of DNA ligases; it exerts its anti-metastatic activity by restraining the $\mathrm{c}$-Src/focal adhesion kinase (FAK)associated signaling pathway in breast cancer cells $(28,31)$ and NC has been shown to inhibit lipopolysaccharide (LPS)induced tumor necrosis factor (TNF)- $\alpha$, interleukin (IL)- $1 \beta$ and IL- 6 production through the suppression of phosphorylation of mitogen-activated protein kinases (MAPKs) and the translocation of p65 (29).

In this study, to further elucidate the effects of $\mathrm{NC}$ and the mechanisms behind its antitumor effects, we investigated the effect of $\mathrm{NC}$ on the apoptosis and proliferation of HCC cells using a mouse xenograft model of HCC and investigated the possible molecular mechanisms mediating its biological effects.

\section{Materials and methods}

Materials and reagents. Dulbecco's modified Eagle's medium (DMEM), fetal bovine serum (FBS), penicillin-streptomycin, trypsin-EDTA, TRIzol reagent and iBlot Western detection stack/iBlot dry blotting system were purchased from Invitrogen (Grand Island, NY, USA). Superscript II reverse transcriptase was provided by Promega (Madison, WI, USA). Bcl-2, Bax, CDK4, cyclin D1, p21, JAK1, STAT3 antibodies and horseradish peroxidase (HRP)-conjugated secondary antibodies were obtained from Cell Signaling Technology, Inc. (Beverly, MA, USA). Proliferating cell nuclear antigen (PCNA) assay and terminal deoxynucleotidyl transferase-mediated dUTP nick end-labeling (TUNEL) assay kits were purchased from R\&D Systems (Minneapolis, MN, USA), the Vectastain ${ }^{\circledR}$ Elite ABC kit was provided by Vector Laboratories, Inc. (Burlingame,
CA, USA). All other chemicals used, unless otherwise stated, were obtained from Sigma-Aldrich (St. Louis, MO, USA).

Drugs. NC was provided by the Sichuan Institute of Biochemical Technology, Chengdu, China and the purity was determined to be $98 \%$ by high-performance liquid chromatography (HPLC). A stock solution of NC was prepared in dimethyl sulfoxide (DMSO; Sigma-Aldrich). Aliquots were stored at $-20^{\circ} \mathrm{C}$.

Cell culture. HepG2 HCC cells were obtained from the American Type Culture Collection (ATCC; Manassas, VA, USA). The cells were grown in DMEM containing $10 \%(\mathrm{v} / \mathrm{v})$ FBS and $100 \mathrm{U} / \mathrm{ml}$ penicillin and $100 \mu \mathrm{g} / \mathrm{ml}$ streptomycin in a $37^{\circ} \mathrm{C}$ humidified incubator with $5 \% \mathrm{CO}_{2}$.

Animals. Sixteen nude mice (with an initial body weight of 20-22 g) were provided by the Shanghai Laboratory Animal Center, Shanghai, China and all animal experiments were carried out strictly in accordance with the international ethics guidelines and the National Institutes of Health Guide concerning the Care and Use of Laboratory Animal and the experiments were approved by the Ethics Committee of Fujian University of Traditional Chinese Medicine, Fuzhou, China. All mice were maintained under a controlled environment [temperature $\left(21 \pm 2^{\circ} \mathrm{C}\right)$, humidity $(50 \pm 10 \%)$ and a $12 \mathrm{~h} \mathrm{light} /$ dark cycle] with free access to food and tap water. Cells $\left(1.5 \times 10^{6}\right)$ mixed with Matrigel (1:1) were inoculated subcutaneously into the right inguinal region of the mice. The administration began 3 days after inoculation. The mice were divided into 2 groups: the control group (daily intraperitoneal injection of saline) and the NC group (daily intraperitoneal injection of NC $10 \mathrm{mg} /$ $\mathrm{kg}$ ); each group comprised 8 mice, including 4 males and 4 females. Every 2 days, individual mice were weighed and each liver tumor was measured using calipers. After 14 days, the mice were euthanized using $100 \mathrm{mg} / \mathrm{kg}$ of pentobarbital sodium (Nembutal; Boehringer Ingelheim, Artarmon, New South Wales, Australia), and the tumor tissue was removed and weighed. Tumor growth was determined by measuring the major (L) and minor (W) diameter with a caliper. The tumor volume was calculated according to the following formula: tumor volume $=\pi / 6 \times \mathrm{L} \mathrm{x} \mathrm{W}^{2}$.

TUNEL assay for apoptosis. Tumor samples were fixed in $4 \%$ buffered paraformaldehyde for $12 \mathrm{~h}$, and subsequently processed conventionally for paraffin-embedded tumor sections ( $4 \mu \mathrm{m}$ thick). The tumor sections were then deparaffinized and rehydrated by treatment with a series of xylenes and graded alcohols. Tissue sections from paraffin-embedded tumors were examined by TUNEL assay for the quantitative analyses of apoptosis. TUNEL assay was performed using an In Situ Cell Death Detection kit (R\&D Systems). Apoptotic cells were counted as DAB-positive cells (brown stained) at 5 arbitrarily selected microscopic fields at a magnification of $\mathrm{x} 400$. TUNEL-positive cells were counted as a percentage of the total cells.

Immunohistochemistry (IHC). Tumor samples were collected after processing as described above. The endogenous peroxidase activity of the sections was quenched by incubating in water containing $0.3 \% \mathrm{H}_{2} \mathrm{O}_{2}$ for $30 \mathrm{~min}$. IHC staining was 
performed using the Vectastain Elite $\mathrm{ABC}$ kit according to the manufacturer's instructions. Briefly, after blocking nonspecific proteins with normal serum in PBS (0.1\% Tween-20), the sections were treated with PCNA, Bcl-2, Bax, CDK4, cyclin D1 and p21 antibody (at a dilution of 1:200). The sections were incubated with a biotinylated anti-rabbit IgG antibody for $30 \mathrm{~min}$ and then treated with the $\mathrm{ABC}$ reagent for $30 \mathrm{~min}$. They were finally treated with $\mathrm{DAB}$ for $10 \mathrm{~min}$. After the sections were washed and air-dried, cover slips were applied to the sections using permount slide mounting medium.

All indicators were quantified by counting respective positive cells and total number of cells in 5 high-power fields (x400) randomly selected in each slide, and the average proportion of positive cells in each field was counted using the true color multi-functional cell image analysis management system (Image-Pro Plus; Media Cybernetics, Bethesda, MD, USA).

Western blot analysis. Six tumors were selected randomly from the control or NC group, homogenized in non-denaturing lysis buffer using homogenizer and centrifuged at 15,000 x g for $15 \mathrm{~min}$ followed by the determination of protein concentration in the supernatants. Equal protein amounts per lysate were separated using NuSep 12\% LongLife Tris Glycine iGels (NuSep Ltd., Lane Cove, New South Wales, Australia) under a reducing condition using $200 \mathrm{~V}$ for $1 \mathrm{~h}$, on Tris-Glycine gels. They were then transferred onto PVDF membranes, and blocked for $2 \mathrm{~h}$ with $5 \%$ non-fat dry milk. The membranes were incubated with the desired primary antibody to JAK1, p-JAK1, STAT3, p-STAT3 and $\beta$-actin (at a dilution of 1:1,000) overnight at $4^{\circ} \mathrm{C}$ and then with appropriate HRP-conjugated secondary antibody followed by enhanced chemiluminescence detection.

Statistical analysis. All data are presented as the means \pm SD. Statistical calculations were performed using the SPSS package for Windows (version 17.0). Statistical analysis of the data was performed using the Student's t-test and ANOVA. P-values $<0.05$ were considered to indicate statistically significant differences.

\section{Results}

NC inhibits tumor growth in HCC tumor xenografts in mice. To evaluate the antitumor effect of $\mathrm{NC}$, the tumor volume and weight were measured in HCC tumor xenografts in mice, and the side-effect were determined based on the measurement of body weight gain. In Fig. 1A and B, tumor volume and weight in the NC group were reduced by 52 and $41 \%$, respectively, as compared to the tumors from the control mice. Nevertheless, $\mathrm{NC}$ treatment exerted no effect on the body weight gain in the experimental animals (Fig. 1C). These data indicate that NC inhibits HCC tumor growth in vivo, without evident signs of toxicity.

NC induces apoptosis and inhibits cell proliferation in HCC tumor xenografts in mice. The effect of NC on apoptosis and cell proliferation in $\mathrm{HCC}$ tumor xenografts in mice was examined by TUNEL assay and IHC staining for PCNA. The number of TUNEL-positive cells was significantly lower in the tumors from the NC-treated group as compared to those from
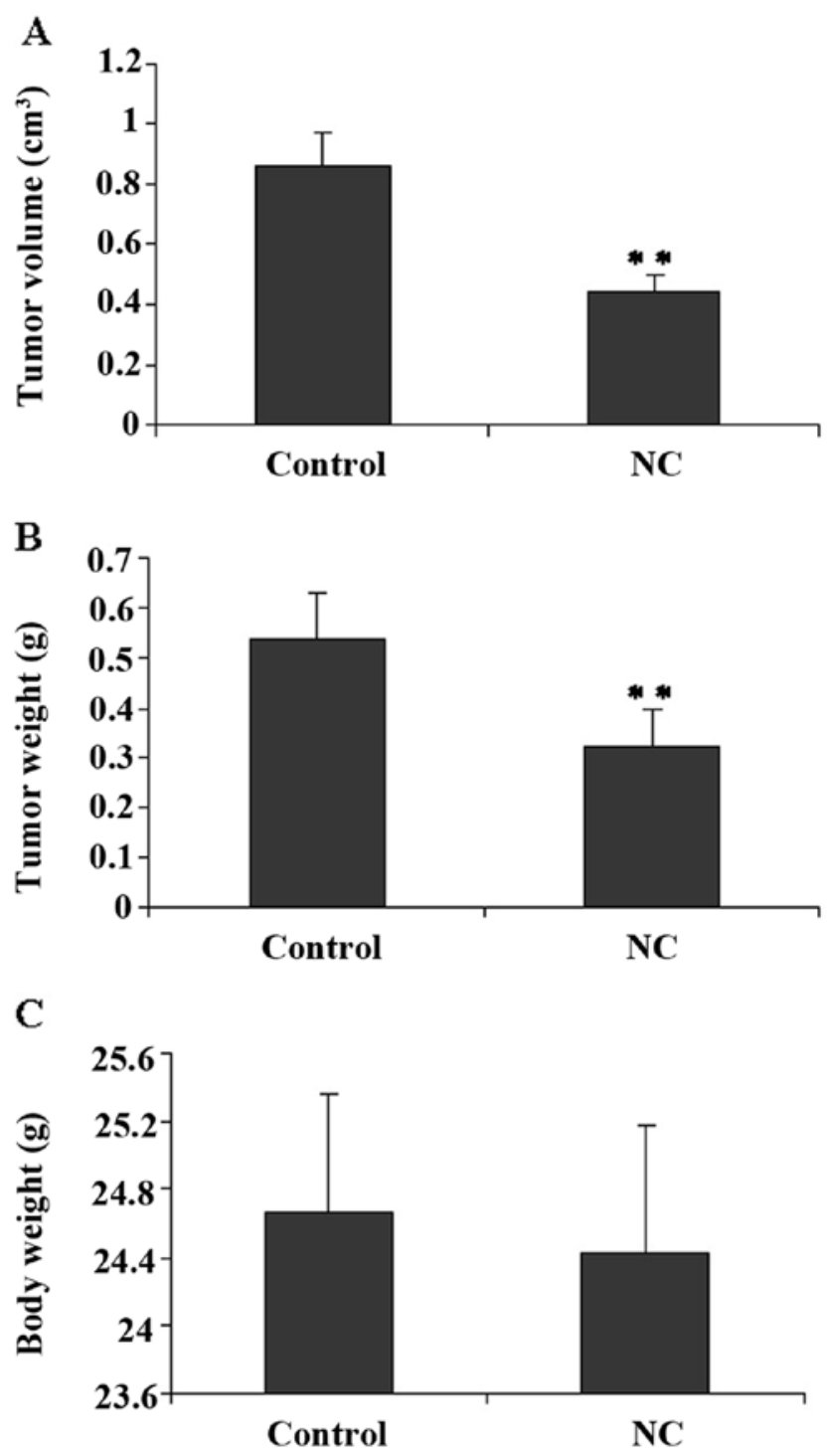

Figure 1. Effect of nitidine chloride (NC) on tumor growth in hepatocellular carcinoma (HCC) tumor xenografts in mice. After tumor development, the mice were intraperitoneally injected with a $10 \mathrm{mg} / \mathrm{kg} /$ day dose of NC or saline daily for 14 days. (A) Tumor volume, (B) tumor weight and (C) body weight were examined at the end of the experiment. Data shown are the means \pm SD (error bars) $(\mathrm{n}=8) .{ }^{*} \mathrm{P}<0.05$ and ${ }^{* *} \mathrm{P}<0.01$ compared to the control group.

the control mice (Fig. 2A-C). The number of PCNA-positive cells in the control group was markedly higher than that in the NC-treated mice (Fig. 2D-F). These results suggest that NC induces apoptosis and inhibits HepG2 cell growth in vivo.

NC inhibits the JAK1-STAT3 signaling pathway in HCC tumor xenografts in mice. It is well known that STAT3 proteins induce the phosphorylation of JAK tyrosine kinase. Therefore, in this study, we examined the effect of NC on JAK1 and STAT3 phosphorylation at $\mathrm{Tyr}^{705}$ in tumor tissue by western blot analysis. Fig. 3 illustrates that JAK1 and STAT3 protein phosphorylation in the NC-treated group was lower than that in the control mice, while the level of JAK1 and STAT3 remained unaltered following treatment with $\mathrm{NC}$, indicating that NC evidently inhibited the JAK and STAT3 activation in vivo. 

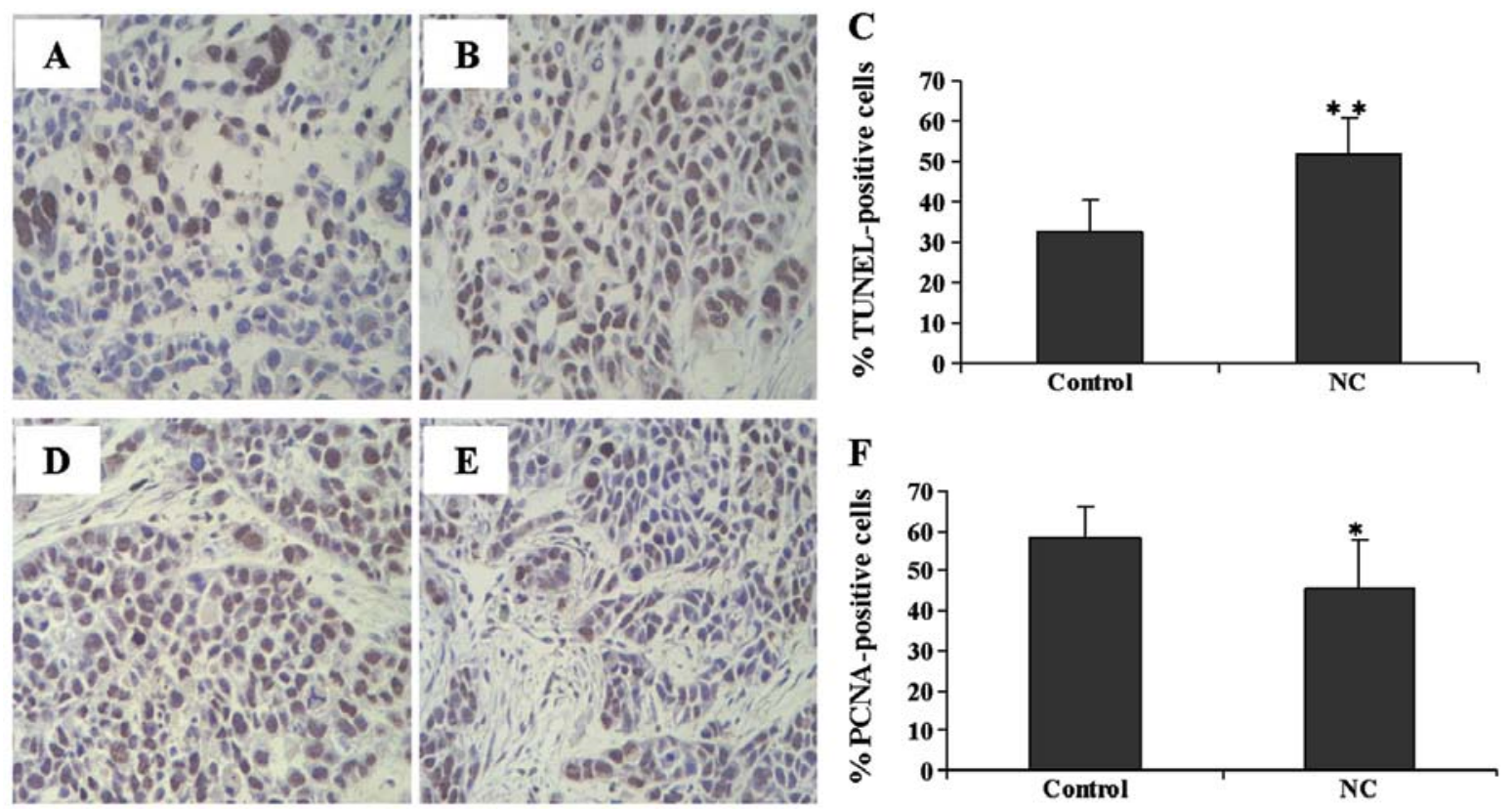

Figure 2. Effect of nitidine chloride (NC) on cell apoptosis and proliferation in hepatocellular carcinoma (HCC) tumor xenografts in mice. At the end of the experiment, tumor tissues were prepared for immunohistochemical (IHC) staining for TUNEL and PCNA. (A) TUNEL control group, (B) TUNEL NC-treated group, (D) PCNA control group, and (E) PCNA NC-treated group. (C and F) Quantification of IHC assay is represented as percentage of positively-stained cells of (C) TUNEL and (F) PCNA. The columns represent the means $\pm \mathrm{SD}$ (error bars) $(\mathrm{n}=8)$. ${ }^{*} \mathrm{P}<0.05$ and ${ }^{* *} \mathrm{P}<0.01$ compared to the control group.

A
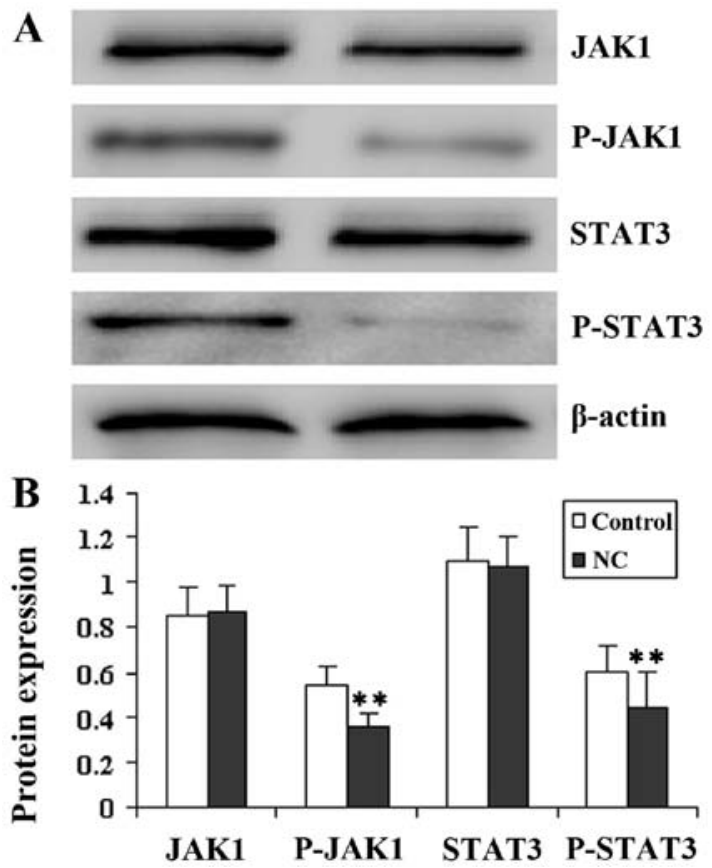

Figure 3. Effect of nitidine chloride (NC) on the phosphorylation of JAK1 and STAT3 in hepatocellular carcinoma (HCC) tumor xenografts in mice. (A) The levels of JAK1 and STAT3 phosphorylation in the tumor tissues were determined by western blot analysis using an antibody that recognizes phosphorylated JAK1 and STAT3 at $\mathrm{Tyr}^{705}$. $\beta$-actin was used as the internal control for western blot analysis. The blots are representative of 8 specimens. (B) Densitometric analysis. Data shown are the means \pm SD (error bars) $(n=8) .{ }^{*} \mathrm{P}<0.05$ and ${ }^{* *} \mathrm{P}<0.01$ compared to the control group.

$N C$ regulates the expression of $B c l-2, B a x, C D K 4$, cyclin $D 1$ and 21 in HCC tumor xenografts in mice. To further investigate the mechanism behind the pro-apoptotic and anti- proliferative effects of $\mathrm{NC}$, we examined the protein expression of Bcl-2, Bax, CDK4, cyclin D1 and p21. The results from immunohistochemistry analysis (Fig. 4) showed that NC treatment markedly reduced the expression of anti-apoptotic Bcl-2, pro-proliferative cyclin D1 and CDK4 proteinm but increased that of pro-apoptotic Bax and anti-proliferative p21 in the HCC tumor xenografts in mice compared to the control mice.

\section{Discussion}

Cancer cells are characterized by an uncontrolled increase in cell proliferation and/or a reduction in cell apoptosis, which not only confers a survival advantage to the cancer cells but also causes resistance to conventional chemotherapeutic agents $(32,33)$. The currently used anticancer chemotherapetuic drugs have several side-effects on normal cells, thus limiting their long-term use. However, natural products with fewer side-effects, have long been used in Chinese medicine for the treatment of various types of cancer, including liver cancer, and these products have shown pro-apoptotic activity and may thus be promising approach to cancer therapy. Zanthoxylum nitidum, a well-known traditional Chinese folk medicine, is used to promote the flow of Qi, relieve pain, promote blood circulation and to remove blood stasis (27). However, the precise mechanism behind its potential antitumor activity has not yet been fully elucidated. Therefore, the antitumor activity of NC and the underlying molecular mechanisms require further study before it can be further developed as an anticancer agent.

Mitochondria-bound Bcl-2 and Bax proteins play a crucial role in the control of apoptosis. Bcl-2 family proteins are key regulators of apoptosis, functioning as either promoters (Bax), or suppressors (Bcl-2). Lower Bax to $\mathrm{Bcl}-2$ ratios due to the 

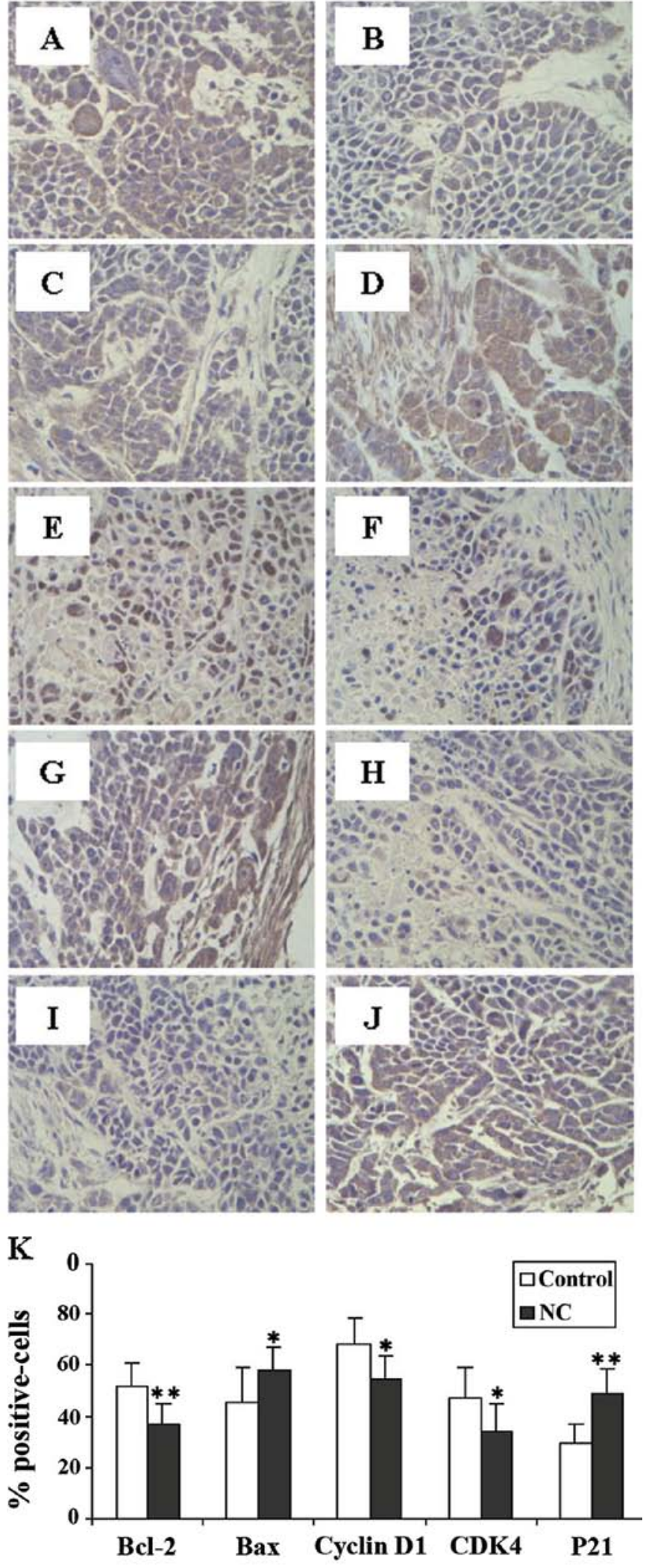

Figure 4. Effect of nitidine chloride (NC) on the expression of Bcl-2, Bax, cyclin D1, CDK4 and p21 in hepatocellular carcinoma (HCC) tumor xenografts in mice. The images are representative images taken at a magnification of $\mathrm{x} 400$, and the expression of these tumor tissues was determined by immunohistochemistry. (A) Bcl-2, (C) Bax, (E) cyclin D1, (G) CDK4 and (I) p21 in the control group; (B) Bcl-2, (D) Bax, (F) cyclin D1, (H) CDK4 and (J) p21 in the NC-treaded group. (K) Quantification of immunohistochemical assay is represented as the percentage of positively-stained cells. Data shown are the means $\pm \mathrm{SD}$ (error bars) $(\mathrm{n}=8) .{ }^{*} \mathrm{P}<0.05$ and ${ }^{* *} \mathrm{P}<0.01$ compared to the control group. downregulation of Bax expression or the overexpression of Bcl-2 are commonly observed in cancer cells (32,34-36). In the present study, we demonstrated that NC inhibited tumor growth and enhanced Bax expression, but reduced Bcl-2 expression in HCC tumor xenografts in mice.

Eukaryotic cell proliferation is primarily regulated by the cell cycle. The orderly progression of the cell cycle is affected through the periodic activation and inactivation of a series of CDKs (37). G1/S checkpoints are located at the end of the G1 phase of the cell cycle, just before entry into the $\mathrm{S}$ phase. The CDK4/cyclin D complex phosphorylates the retinoblastoma $(\mathrm{Rb})$ protein, promoting cyclin E expression through its E2F-dependent transcriptional activation and the disruption of the Rb-histone deacetylase (HDAC) complex, culminating in CDK2 kinase activation as the $\mathrm{S}$ phase progresses $(38,39)$. Uncontrolled cell division and malignancy occur due to an unchecked or hyper-activated cyclin D1/CDK4 complex. Normal p21 function plays a crucial role in the induction of apoptosis and cell cycle G1/S transition checkpoints in human and murine cells following DNA damage (40), and the downregulation of $\mathrm{p} 21$ expression is associated with the promotion of tumor formation and a poor prognosis in many types of cancer (41). In this study, we found that NC downregulated the expression of cyclin D1/CDK4 and upregulated the expression of p21 in vivo, suggesting that $\mathrm{NC}$ inhibited cancer cell proliferation by inducing arrest at the G1/S phase.

As one of the more recently recognized oncogenic signaling pathways, the JAK1-STAT3 signaling pathway has been shown to be important for tumorigenesis. The persistent activation of JAK1/STAT3 signaling contributes to the malignancy of tumors by promoting tumor cell proliferation and survival, angiogenesis and immune evasion $(10,34,42,43)$. Thus, the JAK1-STAT3 signaling pathway is a promising molecular target in cancer therapy. Numerous studies have revealed that STAT3 contributes to oncogenesis through several mechanisms, including the inhibition of apoptosis, the enhancement of cell proliferation, the induction of angiogenesis and the suppression of immune responses $(24,44)$. Our study demonstrates that NC blocks the phosphorylation of JAK1-STAT3 in $\mathrm{HCC}$ tumor xenografts in mice.

In conclusion, in the present study, we demonstrate that $\mathrm{NC}$ inhibits tumor growth in HCC tumor xenografts in mice. In addition, our results revealed that $\mathrm{NC}$ blocked the activation of the JAK1-STAT3 pathway. Consequently, the inhibitory effect of NC on JAK1-STAT3 activation led to the induction of cell apoptosis which resulted from the decrease in the $\mathrm{Bcl}-2 / \mathrm{Bax}$ ratio, and the suppression of cell proliferation which originated from G1/S phase arrest through the decrease in CDK4 and cyclin D1 expression and the increase in p21 expression.

\section{Acknowledgements}

The study was supported by grants from the National Natural Science Foundation of China (nos. 81273836 and 81001554).

\section{References}

1. Ferenci P, Fried M, Labrecque D, et al: Hepatocellular carcinoma (HCC): a global perspective. J Clin Gastroenterol 44: 239-245, 2010. 
2. Cheng AL, Kang YK, Chen Z, et al: Efficacy and safety of sorafenib in patients in the Asia-Pacific region with advanced hepatocellular carcinoma: a phase III randomised, double-blind, placebo-controlled trial. Lancet Oncol 10: 25-34, 2009.

3. Bridges JF, Dong L, Gallego G, Blauvelt BM, Joy SM and Pawlik TM: Prioritizing strategies for comprehensive liver cancer control in Asia: a conjoint analysis. BMC Health Serv Res 12: 376, 2012.

4. Wörns MA and Galle PR: Future perspectives in hepatocellular carcinoma. Dig Liver Dis 42 (Suppl 3): S302-S309, 2010.

5. Jemal A, Bray F, Center MM, Ferlay J, Ward E and Forman D: Global cancer statistics. CA Cancer J Clin 61: 69-90, 2011.

6. Bruix J, Sherman M, Llovet JM, et al: Clinical management of hepatocellular carcinoma. Conclusions of the Barcelona-2000 EASL conference. European Association for the Study of the Liver. J Hepatol 35: 421-430, 2001.

7. Levin B and Amos C: Therapy of unresectable hepatocellular carcinoma. N Engl J Med 332: 1294-1296, 1995.

8. Abou-Alfa GK, Huitzil-Melendez F-D, O'Reilly EM and Saltz LB: Current management of advanced hepatocellular carcinoma. Gastrointest Cancer Res 2: 64-70, 2008.

9. Boos G and Stopper H: Genotoxicity of several clinically used topoisomerase II inhibitors. Toxicol Lett 116: 7-16, 2000.

10. Auyeung KK and Ko JK: Coptis chinensis inhibits hepatocellular carcinoma cell growth through nonsteroidal anti-inflammatory drug-activated gene activation. Int J Mol Med 24: 571-577, 2009.

11. Gordaliza M: Natural products as leads to anticancer drugs. Clin Transl Oncol 9: 767-776, 2007.

12. Newman DJ, Cragg GM and Snader KM: The influence of natural products upon drug discovery. Nat Prod Rep 17: 215-234, 2000.

13. Yamaoka K, Saharinen P, Pesu M, Holt VE III, Silvennoinen O and O'Shea JJ: The Janus kinases (Jaks). Genome Biol 5: 253 , 2004.

14. Aaronson DS and Horvath CM: A road map for those who don't know JAK-STAT. Science 296: 1653-1655, 2002.

15. O'Shea JJ, Gadina M and Schreiber RD: Cytokine signaling in 2002: new surprises in the Jak/Stat pathway. Cell 109 (Suppl): S121-S131, 2002.

16. Darnell JE Jr, Kerr IM and Stark GR: Jak-STAT pathways and transcriptional activation in response to IFNs and other extracellular signaling proteins. Science 264: 1415-1421, 1994.

17. Kisseleva T, Bhattacharya S, Braunstein J and Schindler C: Signaling through the JAK/STAT pathway, recent advances and future challenges. Gene 285: 1-24, 2002.

18. Liu L, Nam S, Tian Y, et al: 6-Bromoindirubin-3'-oxime inhibits JAK/STAT3 signaling and induces apoptosis of human melanoma cells. Cancer Res 71: 3972-3979, 2011.

19. Bromberg J and Darnell JE Jr: The role of STATs in transcriptional control and their impact on cellular function. Oncogene 19: 2468-2473, 2000.

20. Xu Q, Briggs J, Park S, et al: Targeting Stat3 blocks both HIF-1 and VEGF expression induced by multiple oncogenic growth signaling pathways. Oncogene 24: 5552-5560, 2005.

21. Liao J, Ke M, Xu T and Lin L: Electroacupuncture inhibits apoptosis in annulus fibrosis cells through suppression of the mitochondria-dependent pathway in a rat model of cervical intervertebral disc degradation. Genet Mol Biol 35: 686-692, 2012.

22. Yarani R, Mansouri K, Mohammadi-Motlagh HR, Mahnam A and Emami Aleagha MS: In vitro inhibition of angiogenesis by hydroalcoholic extract of oak (Quercus infectoria) acorn shell via suppressing VEGF, MMP-2, and MMP-9 secretion. Pharm Biol 51: 361-368, 2012.

23. Chen J, Wang J, Lin L, et al: Inhibition of STAT3 signaling pathway by nitidine chloride suppressed the angiogenesis and growth of human gastric cancer. Mol Cancer Ther 11: 277-287, 2012 .
24. Buettner R, Mora LB and Jove R: Activated STAT signaling in human tumors provides novel molecular targets for therapeutic intervention. Clin Cancer Res 8: 945-954, 2002.

25. Calvisi DF, Pascale RM and Feo F: Dissection of signal transduction pathways as a tool for the development of targeted therapies of hepatocellular carcinoma. Rev Recent Clin Trials 2: 217-236, 2007.

26. Imada $\mathrm{K}$ and Leonard WJ: The Jak-STAT pathway. Mol Immunol 37: 1-11, 2000.

27. Bencao Z (ed): Chinese Herbal Medicine. Chinese Edition. Shanghai Science and Technology Publishing House, Shanghai, 1996.

28. Pan X, Han H, Wang L, et al: Nitidine chloride inhibits breast cancer cells migration and invasion by suppressing c-Src/FAK associated signaling pathway. Cancer Lett 313: 181-191, 2011.

29. Wang Z, Jiang W, Zhang Z, Qian M and Du B: Nitidine chloride inhibits LPS-induced inflammatory cytokines production via MAPK and NF-kappaB pathway in RAW 264.7 cells. J Ethnopharmacol 144: 145-150, 2012.

30. Liu LM and Liu HG: Anti-hepatoma activity of nitidine chloride and its effect on topoisomerase. Chin Pharmacol Bull 4: 497-500, 2010 (In Chinese).

31. Cushman M, Mohan P and Smith EC: Synthesis and biological activity of structural analogs of the anticancer benzophenanthridine alkaloid nitidine chloride. J Med Chem 27: 544-547, 1984

32. Adams J and Cory S: The Bcl-2 apoptotic switch in cancer development and therapy. Oncogene 26: 1324-1337, 2007.

33. Cai Q, Lin J, Wei L, et al: Hedyotis diffusa Willd inhibits colorectal cancer growth in vivo via inhibition of STAT3 signaling pathway. Int J Mol Sci 13: 6117-6128, 2012.

34. Hall C, Troutman SM, Price DK, Figg WD and Kang MH: $\mathrm{Bcl}-2$ family of proteins as therapeutic targets in genitourinary neoplasms. Clin Genitourin Cancer 11: 10-19, 2013.

35. Paul-Samojedny M, Kokocińska D, Samojedny A, et al: Expression of cell survival/death genes: Bcl-2 and Bax at the rate of colon cancer prognosis. Biochim Biophys Acta 1741: 25-29, 2005.

36. Youle RJ and Strasser A: The BCL-2 protein family: opposing activities that mediate cell death. Nat Rev Mol Cell Biol 9: 47-59, 2008.

37. Chae HD, Kim J and Shin DY: NF-Y binds to both G1- and G2-specific cyclin promoters; a possible role in linking CDK2/ Cyclin A to CDK1/Cyclin B. BMB Rep 44: 553-557, 2011.

38. Sherr CJ: Cancer cell cycles. Science 274: 1672-1677, 1996.

39. Tin AS, Sundar SN, Tran KQ, Park AH, Poindexter KM and Firestone GL: Antiproliferative effects of artemisinin on human breast cancer cells requires the downregulated expression of the E2F1 transcription factor and loss of E2F1-target cell cycle genes. Anticancer Drugs 23: 370-379, 2012.

40. Zafonte BT, Hulit J, Amanatullah DF, et al: Cell-cycle dysregulation in breast cancer: breast cancer therapies targeting the cell cycle. Front Biosci 5: D938-D961, 2000.

41. Domagala W, Welcker M, Chosia M, et al: p21/WAF1/Cip1 expression in invasive ductal breast carcinoma: relationship to p53, proliferation rate, and survival at 5 years. Virchows Arch 439: 132-140, 2001

42. Whang-Peng J, Cheng AL, Hsu C and Chen CM: Clinical development and future direction for the treatment of hepatocellular carcinoma. J Exp Clin Med 2: 93-103, 2010.

43. Fracchiolla N,Capaccio P, Carboni N, et al: Immunohistochemical and molecular analysis of bax, bcl-2 and p53 genes in laryngeal squamous cell carcinomas. Anticancer Res 19: 1043-1051, 1999.

44. Diaz N, Minton S, Cox C, et al: Activation of stat3 in primary tumors from high-risk breast cancer patients is associated with elevated levels of activated SRC and survivin expression. Clin Cancer Res 12: 20-28, 2006. 